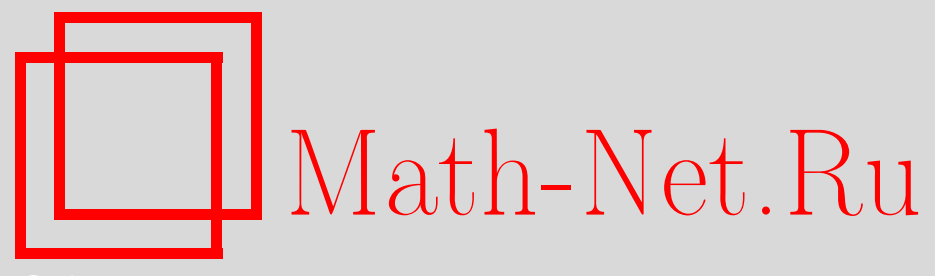

И. А. Круглов, О гомоморфизмах сильно связных конечных автоматов на подстановочные автоматы, Матем. вопр. криптогр., 2011, том 2, выпуск 3, 31-46

DOI: https://doi.org/10.4213/mvk35

Использование Общероссийского математического портала Math-Net.Ru подразумевает, что вы прочитали и согласны с пользовательским соглашением http: //www.mathnet.ru/rus/agreement

Параметры загрузки:

IP : 18.234 .197 .8

26 апреля 2023 г., 13:33:20 
УДК: 519.713 .1

\title{
О гомоморфизмах сильно связных конечных автоматов на подстановочные автоматы
}

\author{
И. А. Круглов \\ ООО «Центр сертификационных исследований», Москва
}

Получено 10.V.2011

Рассмотрен подход к описанию гомоморфизмов сильно связных автоматов на подстановочные автоматы, использующий специальные гомоморфизмы, при композиции которых с гомоморфизмом исходного автомата на подстановочный автомат сохраняется группа автомата-образа. На основе этого подхода построен критерий наличия у сильно связного автомата нетривиального подстановочного гомоморфного образа, у которого частичные функции переходов порождают коммутативную группу подстановок.

Ключевые слова: конечный автомат, гомоморфизм

On the homomorphisms of strongly connected finite automata into substitution automata

\section{A. Kruglov}

\section{LLC Certification Research Center, Moscow}

Abstract. An approach to a description of homomorphisms of strongly connected finite automata into substitution automata is suggested. This approach is based on special homomorphisms such that their composition with homomorphism of an automaton considered into substitution automaton preserves the group of an image. By means of this approach for strongly connected automata a criterion is given for the existence of nontrivial substitutional homomorphic image wish commutative partial transition functions.

Key words: finite automata, homomorphism

Citation: Mathematical Aspects of Cryptography, 2011, vol. 2, no. 3, pp. 31-46 (Russian). 
В настоящей работе используются основные понятия теории автоматов из книги [2]. Следуя [3], под подстановочным автоматом мы понимаем автомат, все частичные функции перехода которого являются подстановками алфавита состояний. Рассмотрим один из подходов к описанию гомоморфизмов конечных сильно связных автоматов на подстановочные автоматы без выхода, мотивированный следующим наблюдением.

Предположим, что заданы конечные автоматы без выхода $A=$ $=\left(X_{A}, S_{A}, \delta_{A}\right), B=\left(X_{B}, S_{B}, \delta_{B}\right)$, где $X_{A}, X_{B}-$ входные алфавиты, $S_{A}, S_{B}-$ алфавиты состояний, $\delta_{A}, \delta_{B}-$ функции переходов:

$$
\delta_{A}: S_{A} \times X_{A} \rightarrow S_{A}, \quad \delta_{B}: S_{B} \times X_{B} \rightarrow S_{B} .
$$

Будем предполагать, что автомат $A$ сильно связный, частичные функции переходов автомата $B$

$$
\delta_{B}^{(y)}: S_{B} \rightarrow S_{B}, \delta_{B}^{(y)}(s)=\delta_{B}(s, y), \forall s \in S_{B}, \forall y \in X_{B},
$$

попарно различны. Предположим также, что пара отображений

$$
\varphi=(\alpha, \beta), \quad \alpha: X_{A} \rightarrow X_{B}, \quad \beta: S_{A} \rightarrow S_{B},
$$

есть гомоморфизм автомата $A$ на автомат $B$. Это означает, что отображения $\alpha, \beta$ сюръективны и что выполнено соотношение

$$
\delta_{B}(\beta(s), \alpha(x))=\beta\left(\delta_{A}(s, x)\right), \forall x \in X_{A}, \forall s \in S_{A} .
$$

Отображение $\alpha$ определяет отображение

$$
f_{\alpha}: X_{A} \rightarrow G_{B}, f_{\alpha}(x)=\delta_{B}^{(\alpha(x))}, \forall x \in X_{A},
$$

множества $X_{A}$ в полугруппу преобразований $\left(G_{B}, \cdot\right)$ множества $S_{B}$, порожденную частичными функциями переходов $\delta_{B}^{(y)}, y \in X_{B}$, автомата $B$ (т.е. в полугруппу автомата $B$ ) с операцией " . ” произведения отображений:

$$
(g \cdot h)(s)=h(g(s)), \forall g, h \in G_{B}, \forall s \in S_{B} .
$$

Зафиксируем произвольное состояние

$$
s_{0} \in S_{A} .
$$

Утверждение. Гомоморфизм $\varphi$ однозначно определяется отображением $f_{\alpha}$ и образом $\beta\left(s_{0}\right)$. 
Доказательство. Для любого $x \in X_{A}$ его образ $\alpha(x)$ может быть однозначно определен по преобразованию $f_{\alpha}(x)$ с помощью соотношения (1), так как частичные функции переходов автомата $B$ попарно различны. Так как автомат $A$ сильно связный, то для любого $s \in S_{A}$ существует такая последовательность $x_{1}, x_{2}, \ldots, x_{k}$ элементов алфавита $X_{A}$, что

$$
s=\delta_{A}\left(\ldots\left(\delta_{A}\left(\delta_{A}\left(s_{0}, x_{1}\right), x_{2}\right), \ldots\right), x_{k}\right) .
$$

Так как $\varphi-$ гомоморфизм автоматов, то из соотношений (1) и (2) следует равенство

$$
\beta(s)=\left(f_{\alpha}\left(x_{1}\right) \cdot f_{\alpha}\left(x_{2}\right) \cdot \ldots \cdot f_{\alpha}\left(x_{k}\right)\right)\left(\beta\left(s_{0}\right)\right) .
$$

Тем самым утверждение доказано.

В связи с данным наблюдением представляет интерес следующая постановка задачи. Предположим, что заданы сильно связный автомат $A$, некоторая произвольная конечная группа $(G, \cdot)$, такое отображение

$$
f: X_{A} \rightarrow G
$$

что множество $f\left(X_{A}\right)$ является системой образующих элементов группы $G$, и зафиксировано произвольное состояние $s_{0} \in S_{A}$. Построим по отображению $f$ и состоянию $s_{0}$ такой гомоморфизм $\varphi$ автомата $A$ на подстановочный автомат $B$, что группа $G_{B}$ автомата $B$ изоморфна фактор-группе группы $G$ по некоторому ее нормальному делителю $N$.

Для этого автомату $A$ поставим в соответствие граф $\Gamma_{A}=\left(S_{A}, V_{A}\right)$ с множеством вершин $S_{A}$, множество $V_{A}$ ребер которого определяется следующим образом. Для любых вершин $s, t \in S_{A}$, таких, что существует $x \in X_{A}$, для которого $\delta_{A}(s, x)=t$, множество $V_{A}$ содержит пару ребер $v$, ш. Начало ребра $v$ есть вершина $s$, конец ребра $v$ есть вершина $t$, начало ребра w есть вершина $t$, конец ребра ш есть вершина $s$, ребра $v$ и ж имеют взаимнообратную ориентацию. Элементы такой пары $v$, ш далее обозначаются следующим образом: $v=e_{s, t}^{+1}$, $=e_{t, s}^{-1}$. Таким образом, множество $V_{A}$ есть объединение двух непересекающихся подмножеств:

$$
\left\{e_{s, t}^{+1} \mid s, t \in S_{A}, \exists x \in X_{A}: \delta_{A}(s, x)=t\right\}
$$

и

$$
\left\{e_{t, s}^{-1} \mid s, t \in S_{A}, \exists x \in X_{A}: \delta_{A}(s, x)=t\right\} .
$$

Заметим, что в случае, когда для некоторых элементов $s, t \in S_{A}, x, x^{\prime} \in X_{A}$ справедливы равенства $\delta_{A}(s, x)=t, \delta_{A}\left(t, x^{\prime}\right)=s$, ребра $e_{s, t}^{+1}$ и $e_{s, t}^{-1}$ являются 
различными ребрами с одинаковым началом и концом. Данное определение графа $\Gamma_{A}$ соответствует определению графов из пункта 3.2 книги [1].

Непустым путем $z$ в графе $\Gamma_{A}$ называется последовательность ребер вида

$$
z=\left(e_{s_{1}, s_{2}}^{\varepsilon_{1}}, e_{s_{2}, s_{3}}^{\varepsilon_{2}}, \ldots, e_{s_{k}, s_{k+1}}^{\varepsilon_{k}}\right), k \geq 1,
$$

при этом $s_{1}$ назовем началом, а $s_{k+1}-$ концом пути $z$. Если $s_{1}=s_{k+1}$, то путь $z$ вида (3) называется циклом, проходящим через $s_{1}$. Кроме того, для любой вершины $s \in S_{A}$ мы будем рассматривать пустой путь, т. е. путь без ребер, с началом и концом $s$ как пустой цикл $z_{s, s}$ с концом $s$. Обозначим через $Z_{0}$ множество всех циклов в графе $\Gamma_{A}$ с концом $s_{0}$.

Так как автомат $A$ сильно связный, то для любых двух вершин $s, t \in$ $\in S_{A}, s \neq t$, в графе $\Gamma_{A}$ существует путь с началом $s$ и концом $t$. Зафиксируем произвольно такой путь $z_{s, t}$.

Для любых вершин $s, t \in S_{A}$, которые соединены ребром $e_{s, t}^{+1}$, определим множества элементов группы $G$ :

$$
\Omega\left(e_{s, t}^{+1}\right)=\left\{f(x), \delta_{A}(s, x)=t\right\}, \quad \Omega\left(e_{t, s}^{-1}\right)=\left\{f(x)^{-1}, \delta_{A}(s, x)=t\right\} .
$$

Для любого пути $z$ вида (3) введем множество элементов группы $G$ :

$$
\begin{gathered}
\\
\Omega(z)=\Omega\left(e_{s_{1}, s_{2}}^{\varepsilon_{1}}\right) \cdot \Omega\left(e_{s_{2}, s_{3}}^{\varepsilon_{2}}\right) \cdot \ldots \cdot \Omega\left(e_{s_{k}, s_{k+1}}^{\varepsilon_{k}}\right)= \\
=\left\{g_{1} \cdot g_{2} \cdot \ldots \cdot g_{k} \mid g_{j} \in \Omega\left(e_{s_{j}, s_{j+1}}^{\varepsilon_{j}}\right), \forall j \in\{1, \ldots, k\} .\right.
\end{gathered}
$$

Для пустого пути $z$ полагаем $\Omega(z)=\left\{e_{G}\right\}, e_{G}-$ нейтральный элемент группы $G$.

Произвольному пути $z$ вида (3) соответствует путь

$$
z^{-1}=\left(e_{s_{k+1}, s_{k}}^{-\varepsilon_{1}}, e_{s_{k}, s_{k-1}}^{-\varepsilon_{2}}, \ldots, e_{s_{2}, s_{1}}^{-\varepsilon_{1}}\right),
$$

и верно равенство

$$
\Omega\left(z^{-1}\right)=\Omega(z)^{-1} .
$$

Если $s \in S_{A}, z_{1}-$ путь с концом $s, z_{2}$ - путь с началом $s$, то определен путь $z=\left(z_{1}, z_{2}\right)$, полученный приписыванием $z_{2}$ справа от $z_{1}$, и верно равенство

$$
\Omega(z)=\Omega\left(z_{1}\right) \cdot \Omega\left(z_{2}\right) .
$$

Обозначим через

$$
G_{0}^{(f)}=\bigcup_{z \in Z_{0}} \Omega(z)
$$


объединение множеств $\Omega(z)$, соответствующих всевозможным циклам $z$, проходящим через $s_{0}$. Нетрудно видеть, что множество $G_{0}^{(f)}$ замкнуто относительно групповой операции и является подгруппой конечной группы $G$.

Для каждой пары состояний $s, t \in S_{A}$ зафиксируем произвольно элемент $g_{s, t} \in \Omega\left(z_{s, t}\right)$. По определению $g_{s, s}=e_{G}$ для любого $s \in S_{A}$.

Определим автомат $B$ с входным алфавитом и алфавитом состояний:

$$
X_{B}=f\left(X_{A}\right), \quad S_{B}=\left\{G_{0}^{(f)} \cdot g \mid \forall g \in G\right\},
$$

и функцией перехода

$$
\delta_{B}\left(G_{0}^{(f)} \cdot g, y\right)=\delta_{B}^{(y)}\left(G_{0}^{(f)} \cdot g\right)=G_{0}^{(f)} \cdot g \cdot y, \forall G_{0}^{(f)} \cdot g \in S_{B}, \forall y \in X_{B} .
$$

Рассмотрим следующий нормальный делитель группы $G$ :

$$
N=\bigcap_{g \in G} g^{-1} \cdot G_{0}^{(f)} \cdot g .
$$

Теорема 1. 1. Автомат B, определяемый равенствами (7), (8), является подстановочныл, отображения

$$
\begin{gathered}
\alpha(x)=f(x) \quad \forall x \in X_{A}, \\
\beta(s)=G_{0}^{(f)} \cdot g_{s_{0}, s} \quad \forall s \in S_{A}
\end{gathered}
$$

определяют гомоморфизм $\varphi=(\alpha, \beta)$ автомата $A$ на автомат $B$.

2. Группа $G_{B}$ автомата $B$ изоморфна факторгруппе группь $G$ по нормальному делителю $N$, причем существует однозначно определенный изоморфизм групп $\bar{\chi}: G / N \rightarrow G_{B}$, для которого

$$
\bar{\chi}(f(x) \cdot N)=f_{\alpha}(x) \quad \forall x \in X_{A} .
$$

В правой части равенства (12) подстановка $f_{\alpha}(x) \in G_{B}$ определяется соотночениями (1) и (10).

Доказательство. 1. Свойство подстановочности автомата $B$ следует из соотношения (8) и существования обратных элементов в группе $G$. Докажем, что $\varphi-$ гомоморфизм автоматов. Пусть элементы $s, t \in S_{A}, x \in X_{A}$ удовлетворяют соотношению

$$
\delta_{A}(s, x)=t
$$


Надо показать, что верно равенство $\delta_{B}(\beta(s), \alpha(x))=\beta(t)$, т. е., в соответствии с (10) и (11), что

$$
G_{0}^{(f)} \cdot g_{s_{0}, s} \cdot f(x)=G_{0}^{(f)} \cdot g_{s_{0}, t} .
$$

Из соотношения (13) следует, что определен следующий цикл в графе $\Gamma_{A}$, проходящий через $s_{0}$ :

$$
u_{s, t}=\left(z_{s_{0}, s}, e_{s, t}^{+1}, z_{s_{0}, t}^{-1}\right), \quad u_{s, t} \in Z_{0} .
$$

Имеют место включения

$$
g_{s_{0}, s} \cdot f(x) \cdot g_{s_{0}, t}^{-1} \in \Omega\left(u_{s, t}\right), \quad \Omega\left(u_{s, t}\right) \subseteq G_{0}^{(f)},
$$

откуда следует равенство (14).

2. Докажем второе утверждение теоремы. Определим отображение $\chi: G \rightarrow G_{B}$. Рассмотрим произвольный элемент $h \in G$. Так как по предположению относительно отображения $f$ множество $f\left(X_{A}\right)$ является системой образующих элементов группы $G$, то существует последовательность $x_{1}, \ldots, x_{k} \in X_{A}$, для которой

$$
h=f\left(x_{1}\right) \cdot f\left(x_{2}\right) \cdot \ldots \cdot f\left(x_{k}\right) .
$$

Зафиксируем такую последовательность $x_{1}, \ldots, x_{k}$ и положим

$$
\chi(h)=\delta_{B}^{\left(\alpha\left(x_{1}\right)\right)} \cdot \delta_{B}^{\left(\alpha\left(x_{2}\right)\right)} \cdot \ldots \cdot \delta_{B}^{\left(\alpha\left(x_{k}\right)\right)} .
$$

Тогда из соотношений (8) и (10) следует, что для любого $g \in G$ верны равенства

$$
\chi(h)\left(G_{0}^{(f)} \cdot g\right)=G_{0}^{(f)} \cdot g \cdot f\left(x_{1}\right) \cdot f\left(x_{2}\right) \cdot \ldots \cdot f\left(x_{k}\right)=G_{0}^{(f)} \cdot g \cdot h .
$$

В силу соотношения (17) образ $\chi(h)$ не зависит от выбора элементов $x_{1}, \ldots, x_{k} \in X_{A}$. Кроме того, из (17) следует, что $\chi-$ гомоморфизм групп. Образ $\chi(G) \supseteq\left\{\delta_{B}^{(y)} \mid \forall y \in X_{B}\right\}$, следовательно, $\chi$ сюръективно. Используя соотношения (9) и (17), нетрудно показать, что ядро $\operatorname{ker}(\chi)=N$. По теореме о гомоморфизме групп определен изоморфизм $\bar{\chi}: G / N \rightarrow G_{B}$, для которого

$$
\bar{\chi}(h \cdot N)\left(G_{0}^{(f)} \cdot g\right)=G_{0}^{(f)} \cdot g \cdot h, \forall h \in G, \forall G_{0}^{(f)} \cdot g \in S_{B} .
$$

Соотношение (12) следует из последнего равенства при $h=f(x)$ и соотношений (8), (10), (1). 
Единственность изоморфизма $\bar{\chi}$, удовлетворяющего соотношению (12), следует из предположения о том, что множество $f\left(X_{A}\right)$ является системой образующих элементов группы $G$. Теорема 1 доказана.

Отметим, что гомоморфизм $\varphi$, определяемый равенствами (10), (11), не зависит от выбора путей $z_{s_{0}, s}$ и элементов $g_{s_{0}, s} \in \Omega\left(z_{s_{0}, s}\right)$ : если $z_{s}^{\prime}-$ путь в графе $\Gamma_{A}$ с началом $s_{0}$ и концом $s$ и элемент $g_{s}^{\prime} \in \Omega\left(z_{s}^{\prime}\right)$, то $G_{0}^{(f)} \cdot g_{s_{0}, s}=G_{0}^{(f)} \times$ $\times g_{s}^{\prime}$. В качестве совокупности путей $\left\{z_{s_{0}, s}, s \in S_{A}, s \neq s_{0}\right\}$ можно использовать совокупность путей от корня $s_{0}$ до вершин $s \in S_{A}, s \neq s_{0}$, в остовном дереве с корнем $s_{0}$ графа $\Gamma_{A}$, см. [1].

Покажем, что с точностью до изоморфизма автомат $B$ не зависит от выбора состояния $s_{0} \in S_{A}$. Для произвольного фиксированного состояния $s_{1} \in S_{A}$ рассмотрим множество $Z_{1}$ всех циклов в графе $\Gamma_{A}$, проходящих через $s_{1}$, а также подгруппу $G_{1}^{(f)}=\bigcup_{z \in Z_{1}} \Omega(z)$ группы $G$. Аналогично соотношениям (7), (8) определим автомат $B_{1}=\left(X_{B_{1}}, S_{B_{1}}, \delta_{B_{1}}\right)$ равенствами

$$
\begin{gathered}
X_{B_{1}}=X_{B}=f\left(X_{A}\right), \quad S_{B_{1}}=\left\{G_{1}^{(f)} \cdot g \mid \forall g \in G\right\}, \\
\delta_{B_{1}}\left(G_{1}^{(f)} \cdot g, y\right)=G_{1}^{(f)} \cdot g \cdot y \quad \forall G_{1}^{(f)} \cdot g \in S_{B_{1}}, \forall y \in X_{B_{1}} .
\end{gathered}
$$

Аналогично соотношениям (10), (11) определим эпиморфизм $\varphi_{1}=\left(\alpha, \beta_{1}\right)$ автомата $A$ на автомат $B_{1}$, где

$$
\beta_{1}(s)=G_{1}^{(f)} \cdot g_{s_{1}, s} \quad \forall s \in S_{A} .
$$

Обозначим через $\mathbf{1}_{f\left(X_{A}\right)}$ тождественное преобразование множества $f\left(X_{A}\right)$ и определим отображение $\lambda: S_{B} \rightarrow S_{B_{1}}$, полагая

$$
\lambda\left(G_{0}^{(f)} \cdot g\right)=G_{1}^{(f)} \cdot g_{s_{1}, s_{0}} \cdot g \quad \forall G_{0}^{(f)} \cdot g \in S_{B}
$$

Далее значок «о» обозначает композицию отображений.

Следствие 1. Пара отображений $\theta=\left(\mathbf{1}_{f\left(X_{A}\right)}, \lambda\right)$ есть изоморфизм автомата $B$ на автомат $B_{1}$, причем эпиморфизм $\varphi_{1}$ есть композиция эпиморфизмов $\varphi$ и $\theta$, m.е. $\beta_{1}=\lambda \circ \beta$.

Доказательство. Имеют место включения

$$
\left(z_{s_{1}, s_{0}}^{-1}, z, z_{s_{1}, s_{0}}\right) \in Z_{0}, \forall z \in Z_{1},\left(z_{s_{1}, s_{0}}, z, z_{s_{1}, s_{0}}^{-1}\right) \in Z_{1}, \forall z \in Z_{0},
$$


откуда с учетом соотношений (6), (18) следуют равенства

$$
\begin{gathered}
G_{0}^{(f)}=g_{s_{1}, s_{0}}^{-1} \cdot G_{1}^{(f)} \cdot g_{s_{1}, s_{0}}, \\
\lambda\left(G_{0}^{(f)} \cdot g\right)=g_{s_{1}, s_{0}} \cdot\left(G_{0}^{(f)} \cdot g\right) \quad \forall G_{0}^{(f)} \cdot g \in S_{B} .
\end{gathered}
$$

Следовательно, отображение $\lambda$ определено корректно. Ввиду биективности левых сдвигов на элементы группы $G$ отображение $\lambda$ биективно. Используя соотношение (18), для любых $G_{0}^{(f)} \cdot g \in S_{B}, y \in X_{B}$ получим цепочку равенств:

$$
\begin{aligned}
\lambda\left(\delta_{B}\left(G_{0}^{(f)} \cdot g, y\right)\right)=\lambda\left(G_{0}^{(f)} \cdot g \cdot y\right)=G_{1}^{(f)} \cdot g_{s_{1}, s_{0}} \cdot g \cdot y= & \\
\quad & =\lambda\left(G_{0}^{(f)} \cdot g\right) \cdot y=\delta_{B_{1}}\left(\lambda\left(G_{0}^{(f)} \cdot g\right), \mathbf{1}_{f\left(X_{A}\right)}(y)\right) .
\end{aligned}
$$

Следовательно, $\psi$ - изоморфизм автоматов. Воспользовавшись соотношениями (10), (18), для любого $s \in S_{A}$ выведем равенства

$$
(\lambda \circ \beta)(s)=\lambda\left(G_{0}^{(f)} \cdot g_{s_{0}, s}\right)=G_{1}^{(f)} \cdot g_{s_{1}, s_{0}} \cdot g_{s_{0}, s}=G_{1}^{(f)} \cdot g_{s_{1}, s}=\beta_{1}(s),
$$

т. е. $\beta_{1}=\lambda \circ \beta$. Следствие 1 доказано.

В связи со следствием 1 произвольный эпиморфизм $\varphi$, определенный для некоторого состояния $s_{0} \in S_{A}$ в п. 1 теоремы 1 , назовем естественным эпиморфизмом, определяемым отображением $f: X_{A} \rightarrow G$.

Будем называть произвольный гомоморфизм автомата $A$ на некоторый автомат $B$ тривиальным, если алфавит состояний $S_{B}$ автомата $B$ состоит из одного элемента: $\left|S_{B}\right|=1$.

Естественный эпиморфизм $\varphi$, определяемый отображением $f: X_{A} \rightarrow G$, тривиален тогда и только тогда, когда подгруппа $G_{0}^{(f)}=G$. Сформулируем эквивалентное условие в терминах порождения группы $G$ некоторым конечным множеством образующих элементов.

Введем обозначение (см. (15))

$$
\Omega=\bigcup_{\substack{s, t \in S_{A} \\ \exists x \in X_{A}: \delta_{A}(s, x)=t}} \Omega\left(u_{s, t}\right) .
$$

Следствие 2. В условиях теоремы 1 естественный эпиморфизм $\varphi$ тривиален тогда и только тогда, когда множество $\Omega$ является системой образуюших элементов группь $G$.

Доказательство. Из соотношений (15), (6) следует, что $\Omega \subseteq G_{0}^{(f)}$. Рассмотрим произвольный проходящий через $s_{0}$ цикл $z$ в графе $\Gamma_{A}$ вида (3), 
здесь $s_{1}=s_{k+1}=s_{0}$. Для множества $\Omega(z)$ верно равенство

$$
\begin{aligned}
& \Omega(z)=\Omega(z)=\left(g_{s_{0}, s_{0}} \cdot \Omega\left(e_{s_{0}, s_{2}}^{\varepsilon_{1}}\right) \cdot g_{s_{0}, s_{2}}^{-1}\right) \cdot\left(g_{s_{0}, s_{2}} \cdot \Omega\left(e_{s_{2}, s_{3}}^{\varepsilon_{2}}\right) \cdot g_{s_{0}, s_{3}}^{-1}\right) \times \\
& \quad \times\left(g_{s_{0}, s_{3}} \cdot \Omega\left(e_{s_{3}, s_{4}}^{\varepsilon_{3}}\right) \cdot \ldots \cdot \Omega\left(e_{s_{k-1}, s_{k}}^{\varepsilon_{k-1}}\right) \cdot g_{s_{0}, s_{k}}^{-1}\right) \cdot\left(g_{s_{0}, s_{k}} \cdot \Omega\left(e_{s_{k}, s_{0}}^{\varepsilon_{k}}\right) \cdot g_{s_{0}, s_{0}}^{-1}\right) .
\end{aligned}
$$

Если для некоторого $i \in\{1, \ldots, k\}$ число $\varepsilon_{i}=-1$, то

$$
g_{s_{0}, s_{i}} \cdot \Omega\left(e_{s_{i}, s_{i+1}}^{-1}\right) \cdot g_{s_{0}, s_{i+1}}^{-1}=\left(g_{s_{0}, s_{i+1}} \cdot \Omega\left(e_{s_{i+1}, s_{i}}^{+1}\right) \cdot g_{s_{0}, s_{i}}^{-1}\right)^{-1}
$$

и существует $x \in X_{A}$, для которого $\delta_{A}\left(s_{i+1}, x\right)=s_{i}$. Поэтому множество $\Omega$ является системой образующих элементов группы $G_{0}^{(f)}$, что и требовалось доказать.

Покажем, что автоматы $B$, определяемые отображениями алфавита $X_{A}$ в конечные группы $G$ с помощью соотношений (7), (8) (т. е. образы при различных естественных эпиморфизмах), являются в некотором смысле «максимальными» подстановочными гомоморфными образами данного сильно связного автомата $A$.

Предположим, что заданы некоторый подстановочный автомат $B^{\prime}=$ $=\left(X_{B^{\prime}}, S_{B^{\prime}}, \delta_{B^{\prime}}\right)$ с попарно различными частичными функциями переходов $\delta_{B^{\prime}}^{\left(y^{\prime}\right)}, y^{\prime} \in X_{B^{\prime}}$ и гомоморфизм

$$
\varphi^{\prime}=\left(\alpha^{\prime}, \beta^{\prime}\right), \quad \alpha^{\prime}: X_{A} \rightarrow X_{B^{\prime}}, \quad \beta^{\prime}: S_{A} \rightarrow S_{B^{\prime}}
$$

автомата $A$ на автомат $B^{\prime}$.

Рассмотрим в качестве группы $G$ группу $\left(G_{B^{\prime}}, \cdot\right)$ автомата $B^{\prime}$. Отображение $\alpha^{\prime}$ определяет отображение $f: X_{A} \rightarrow G_{B^{\prime}}$ :

$$
f(x)=\delta_{B^{\prime}}^{\left(\alpha^{\prime}(x)\right)}, \forall x \in X_{A},
$$

$\left(f=f_{\alpha^{\prime}}\right.$ в обозначениях соотношения (1)). Рассмотрим подстановочный автомат $B$, определяемый автоматом $A$, группой $G=G_{B^{\prime}}$, отображением $f$ и состоянием $s_{0}$ с помощью равенств $(7),(8)$. Пусть $\varphi=(\alpha, \beta)-$ естественный эпиморфизм автомата $A$ на автомат $B$. Определим гомоморфизм $\varphi^{*}=\left(\alpha^{*}, \beta^{*}\right)$ автомата $B$ на автомат $B^{\prime}$.

Согласно (7) алфавит

$$
X_{B}=\left\{\delta_{B^{\prime}}^{\left(\alpha^{\prime}(x)\right)} \mid x \in X_{A}\right\} \subseteq G_{B^{\prime}}
$$


По предположению частичные функции переходов автомата $B^{\prime}$ попарно различны, поэтому если для некоторого элемента $g \in X_{B}$ и элементов $x_{1}, x_{2} \in X_{A}$ верны равенства

$$
g=\delta_{B^{\prime}}^{\left(\alpha^{\prime}\left(x_{1}\right)\right)}=\delta_{B^{\prime}}^{\left(\alpha^{\prime}\left(x_{2}\right)\right)},
$$

то $\alpha^{\prime}\left(x_{1}\right)=\alpha^{\prime}\left(x_{2}\right)$. Следовательно, соотношение

$$
\alpha^{*}(g)=\alpha^{\prime}(x), \text { если } g=\delta_{B^{\prime}}^{\left(\alpha^{\prime}(x)\right)}, \quad x \in X_{A},
$$

корректно определяет биективное отображение $\alpha^{*}: X_{B} \rightarrow X_{B^{\prime}}$.

Воспользуемся обозначениями (4), (5). Так как $\varphi^{\prime}$ - гомоморфизм автоматов, то для любых состояний $s^{(1)}, s^{(2)} \in S_{A}$, любого пути $z$ в графе $\Gamma_{A}$ с началом $s^{(1)}$ и концом $s^{(2)}$ индукцией по числу $k$ ориентированных ребер в представлении $z$ вида (3) можно доказать равенство

$$
\sigma\left(\beta^{\prime}\left(s^{(1)}\right)\right)=\beta^{\prime}\left(s^{(2)}\right), \forall \sigma \in \Omega(z) .
$$

Пусть $s_{0}^{\prime}=\beta^{\prime}\left(s_{0}\right)$. Обозначим через $L^{\prime}$ стабилизатор точки $s_{0}^{\prime}$ в группе подстановок $G_{B^{\prime}}$. Из соотношения (21) для любого цикла $z \in Z_{0}$ и любого элемента $\sigma \in \Omega(z)$ следует равенство $\sigma\left(s_{0}^{\prime}\right)=s_{0}^{\prime}$. Ввиду соотношения (6) отсюда получим включение

$$
G_{0}^{(f)} \subseteq L^{\prime} .
$$

Таким образом, соотношение

$$
\beta^{*}\left(G_{0}^{(f)} \cdot g\right)=g\left(s_{0}^{\prime}\right) \quad \forall G_{0}^{(f)} \cdot g \in S_{B},
$$

корректно определяет отображение $\beta^{*}: S_{B} \rightarrow S_{B^{\prime}}$.

Наконец, определим отображение $\chi:\left\{\delta_{B^{\prime}}^{(y)}, y \in X_{B^{\prime}}\right\} \rightarrow G_{B}$, полагая $\chi\left(\delta_{B^{\prime}}^{(y)}\right)=\delta_{B}^{\left(\delta_{B^{\prime}}^{(y)}\right)}$ для любого $y \in X_{B^{\prime}}$.

Теорема 2. Пара отображений $\varphi^{*}=\left(\alpha^{*}, \beta^{*}\right)$ есть гомоморфизм автомата $B$ на автомат $B^{\prime}$. Гомоморфизм $\varphi^{\prime}$ есть композици гомоморфизмов $\varphi u \varphi^{*}$, m.e.

$$
\begin{aligned}
\alpha^{\prime} & =\alpha^{*} \circ \alpha, \\
\beta^{\prime} & =\beta^{*} \circ \beta .
\end{aligned}
$$

Отображение х может быть продолжено до изоморфизма групп $G_{B^{\prime}} u G_{B}$. 
Доказательство. Докажем, что $\varphi^{*}$ - гомоморфизм автоматов. Пусть элементы $G_{0}^{(f)} \cdot g, G_{0}^{(f)} \cdot h \in S_{B}, y \in X_{B}$ удовлетворяют условию

$$
\delta_{B}\left(G_{0}^{(f)} \cdot g, y\right)=G_{0}^{(f)} \cdot h,
$$

надо обосновать равенство

$$
\delta_{B^{\prime}}\left(\beta^{*}\left(G_{0}^{(f)} \cdot g\right), \alpha^{*}(y)\right)=\beta^{*}\left(G_{0}^{(f)} \cdot h\right) .
$$

Из соотношений (8) и (26) следует, что

$$
G_{0}^{(f)} \cdot g \cdot y=G_{0}^{(f)} \cdot h
$$

Существует $x \in X_{A}$, для которого

$$
y=\delta_{B^{\prime}}^{\left(\alpha^{\prime}(x)\right)}, \alpha^{*}(y)=\alpha^{\prime}(x) .
$$

Используя соотношения (20), (23), (27), (28), получаем цепочку равенств

$$
\begin{aligned}
\delta_{B^{\prime}}\left(\beta^{*}\left(G_{0}^{(f)} \cdot g\right), \alpha^{*}(y)\right) & =\delta_{B^{\prime}}\left(g\left(s_{0}^{\prime}\right), \alpha^{\prime}(x)\right)=\delta_{B^{\prime}}^{\left(\alpha^{\prime}(x)\right)}\left(g\left(s_{0}^{\prime}\right)\right)= \\
& =(g \cdot y)\left(s_{0}^{\prime}\right)=\beta^{*}\left(G_{0}^{(f)} \cdot g \cdot y\right)=\beta^{*}\left(G_{0}^{(f)} \cdot h\right),
\end{aligned}
$$

что и требовалось доказать.

Установим справедливость равенств (24) и (25): из них, в частности, будет следовать сюръективность гомоморфизма $\varphi^{*}$.

В силу соотношений (10) и (19) $\alpha(x)=f(x)=\delta_{B^{\prime}}^{\left(\alpha^{\prime}(x)\right)}$ для любого $x \in X_{A}$. Из этого равенства и соотношения (20) следует:

$$
\alpha^{*}(\alpha(x))=\alpha^{\prime}(x) \quad \forall x \in X_{A},
$$

что доказывает равенство (24).

Согласно равенству (11) для любого $s \in S_{A}$

$$
\beta(s)=G_{0}^{(f)} \cdot g_{s_{0}, s},
$$

где элемент $g_{s_{0}, s} \in \Omega\left(z_{s_{0}, s}\right), z_{s_{0}, s}-$ определенный ранее путь в графе $\Gamma_{A}$ с началом $s_{0}$ и концом $s$. Из соотношения (23) следует, что

$$
\beta^{*}\left(G_{0}^{(f)} \cdot g_{s_{0}, s}\right)=g_{s_{0}, s}\left(s_{0}^{\prime}\right) .
$$


Пусть в соотношении (21) $s^{(1)}=s_{0}, s^{(2)}=s, z=z_{s_{0}, s}, \sigma=g_{s_{0}, s}$, тогда

$$
g_{s_{0}, s}\left(s_{0}^{\prime}\right)=\beta^{\prime}(s) .
$$

Из соотношений (29), (30), (31) следует равенство (25).

Наконец, введенное перед формулировкой данной теоремы отображение $\chi$ является ограничением на множество $\left\{\delta_{B^{\prime}}^{(y)}, y \in X_{B^{\prime}}\right\}$ образующих элементов группы $G_{B^{\prime}}$ эпиморфизма групп $\chi: G_{B^{\prime}} \rightarrow G_{B}$ из доказательства теоремы 1. Автомат $B^{\prime}$, будучи гомоморфным образом сильно связного автомата $A$, сильно связный, группа подстановок $G_{B^{\prime}}$ транзитивна. Из соотношений (9) и (22) следует, что ядро $N$ эпиморфизма $\chi$ есть единичная подгруппа. Таким образом, $\chi-$ изоморфизм групп. Теорема 2 доказана.

При решении прикладных задач представляют интерес условия на заданный автомат $A$, при выполнении которых существует гомоморфизм автомата $A$ на автомат $B$, имеющий в том или ином смысле «простую» структуру. Далее мы установим необходимые и достаточные условия для того, чтобы существовал нетривиальный гомоморфизм заданного автомата $A$ на некоторый подстановочный автомат $B$ с абелевой группой $G_{B}$. Введем дополнительные обозначения.

Рассмотрим некоторое семейство символов

$$
\mathfrak{F}=\left\{\mathfrak{e}_{s, t} \mid s, t \in S_{A}, \exists x \in X_{A}: \delta_{A}(s, x)=t\right\}
$$

и свободную абелеву группу $(\mathfrak{F},+)$, порожденную множеством свободных образующих элементов $\mathfrak{E}$. Определим отображение $\phi$ множества $V_{A}$ ориентированных ребер графа $\Gamma_{A}$ в группу $\mathfrak{f}$ следующим образом. Для любых таких $s, t \in S_{A}$, что существует $x \in X_{A}$, для которого $\delta_{A}(s, x)=t$, полагаем:

$$
\phi\left(e_{s, t}^{+1}\right)=\mathfrak{e}_{s, t}, \phi\left(e_{t, s}^{-1}\right)=-\mathfrak{e}_{s, t} .
$$

Каждому пути $z$ в графе $\Gamma_{A}$ вида (3) соответствует следующий элемент группы $(\mathfrak{F},+)$ :

$$
\phi(z)=\phi\left(e_{s_{1}, S_{2}}^{\varepsilon_{1}}\right)+\phi\left(e_{s_{2}, s_{3}}^{\varepsilon_{2}}\right)+\ldots+\phi\left(e_{s_{k}, s_{k+1}}^{\varepsilon_{k}}\right) .
$$

Обозначим через $\mathfrak{B}$ следующую подгруппу свободной группы $(\mathfrak{f},+)$ :

$$
\mathfrak{P}=\left\{\phi(z) \mid \forall z \in Z_{0}\right\}
$$

(в терминах [1] группа $\mathfrak{B}$ есть образ фундаментальной группы графа $\Gamma_{A}$, рассматриваемой как подгруппа свободной группы, порожденной множеством 
свободных образующих элементов ङ;, при естественном гомоморфизме последней на свободную абелеву группу $(\mathfrak{f},+))$.

Далее, определим бинарное отношение $\rho$ на множестве $X_{A}$ :

$$
\rho=\left\{\left(x, x^{\prime}\right) \mid x, x^{\prime} \in X_{A}, \exists s \in S_{A}: \delta_{A}(s, x)=\delta_{A}\left(s, x^{\prime}\right)\right\} .
$$

Отношение $\rho$ рефлексивно и симметрично, следовательно, его транзитивное замыкание $\bar{\rho}$ является отношением эквивалентности на множестве $X_{A}$. Зафиксируем некоторый набор $\bar{X} \subseteq X_{A}$ представителей классов эквивалентности $\bar{\rho}$. Для любого $x \in X_{A}$ обозначим через $\bar{x}$ представителя класса эквивалентности $\bar{\rho}$, содержащего элемент $x$ :

$$
\bar{x} \in \bar{X}, \quad(x, \bar{x}) \in \bar{\rho} .
$$

Рассмотрим также свободную абелеву группу $\mathfrak{A}$, порожденную множеством свободных образующих элементов $\bar{X}$. Введем отображение $\vartheta:$ : $\rightarrow \bar{X}$, при котором любому символу $\mathfrak{e}_{s, t} \in \mathfrak{F}$ однозначно соответствует элемент $\bar{x}_{s, t} \in \bar{X}$, определяемый соотношением

$$
\bar{x}_{s, t}=\vartheta\left(\mathfrak{e}_{s, t}\right)=\bar{x}, \forall x \in X_{A}: \delta_{A}(s, x)=t .
$$

Указанное отображение $\vartheta$ однозначно продолжается до эпиморфизма свободных абелевых групп $\vartheta: \mathfrak{F} \rightarrow \mathfrak{A}$.

Теорема 3. Нетривиальный гомоморфизм автомата А на некоторый подстановочный автомат $B$ с абелевой группой $G_{B}$ сущеествует тогда и только тогда, когда

$$
\vartheta(\mathfrak{Z}) \neq \mathfrak{A}
$$

Доказательство. Докажем необходимость условия (36) для существования нетривиального гомоморфизма автомата $A$ на некоторый подстановочный автомат $B$ с абелевой группой $G_{B}$. Предположим, что условие (36) не выполняется, т. е.

$$
\vartheta(\mathfrak{Z})=\mathfrak{Q}
$$

По теореме 2 достаточно показать, что для любого отображения $f: X_{A} \rightarrow G$ множества $X_{A}$ в некоторую конечную абелеву группу $(G,+)$ (такого, что множество $f\left(X_{A}\right)$ является системой образующих элементов группы $\left.G\right)$ определяемый этим отображением $f$ с помощью соотношений $(10),(11)$ естественный эпиморфизм тривиален. Зафиксируем $G$ и $f$, для каждого состояния $s_{0} \in S_{A}$ соотношение (6) определяет подгруппу $G_{0}^{(f)}$ группы $G$. Как показано при доказательстве следствия 1 из теоремы 1 , подгруппы множества $\left\{G_{0}^{(f)} \mid s_{0} \in S_{A}\right\}$ 
попарно сопряжены. Так как группа $G$ абелева, то $G_{0}^{(f)}$ не зависит от выбора вершины $s_{0}$ графа $\Gamma_{A}$. В связи с этим далее мы обозначаем эту подгруппу через $G^{(f)}$.

Для таких $s, t \in S_{A}$, что существует $x \in X_{A}$, для которого $\delta_{A}(s, x)=t$, согласно (32) и (35) справедливы равенства

$$
(\vartheta \circ \phi)\left(e_{s, t}^{+1}\right)=\bar{x}_{s, t},(\vartheta \circ \phi)\left(e_{t, s}^{-1}\right)=-\bar{x}_{s, t} .
$$

Из соотношения (16) и условия коммутативности группы $G$ следует, что для таких $x, x^{\prime} \in X_{A}$, что $\left(x, x^{\prime}\right) \in \rho$, верно равенство $f(x)+G^{(f)}=f\left(x^{\prime}\right)+G^{(f)}$, поэтому

$$
f(x)+G^{(f)}=f\left(x^{\prime}\right)+G^{(f)}, \forall x, x^{\prime} \in X_{A}:\left(x, x^{\prime}\right) \in \bar{\rho} .
$$

Рассмотрим ограничение $\bar{f}$ отображения $f$ на множество $\bar{X}$. Отображение $\bar{f}$ однозначно продолжается до гомоморфизма свободной группы $\mathfrak{A}$ в группу $G$; этот гомоморфизм мы также обозначим буквой $\bar{f}$.

Из (35), (38) и (39) следует, что для любого ребра $e_{s, t}^{+1}$ в графе $\Gamma_{A}$

$$
(\bar{f} \circ \vartheta \circ \phi)\left(e_{s, t}^{+1}\right)+G^{(f)}=\Omega\left(e_{s, t}^{+1}\right)+G^{(f)} .
$$

Но тогда вследствие (32), (33), линейности отображений $\vartheta, \bar{f}$ и равенств (5), (6)

$$
(\bar{f} \circ \vartheta \circ \phi)(z)+G^{(f)}=\Omega(z)+G^{(f)}=G^{(f)} \quad \forall z \in Z_{0} .
$$

Таким образом, $(\bar{f} \circ \vartheta \circ \phi)\left(Z_{0}\right) \subseteq G^{(f)}$. Согласно (34) и (37) $(\vartheta \circ \phi)\left(Z_{0}\right)=\mathfrak{A}$, следовательно, $\bar{f}(\mathfrak{A}) \subseteq G^{(f)}$, в частности,

$$
\bar{f}(\bar{X}) \subseteq G^{(f)} .
$$

Из соотношений (39) и (40) получим включение $f\left(X_{A}\right) \subseteq G^{(f)}$. Так как по предположению множество $f\left(X_{A}\right)$ - система образующих элементов группы $G$, то $G^{(f)}=G$, т. е. $f$ определяет тривиальный гомоморфизм, что и требовалось доказать.

Докажем достаточность условия (36) для существования нетривиального гомоморфизма автомата $A$ на некоторый подстановочный автомат $B$ с абелевой группой $G_{B}$.

Предположим, что условие (36) выполнено. Факторгруппа $\mathfrak{A} / \vartheta(\mathfrak{Z})$ есть ненулевая абелева группа с конечным числом образующих элементов, 
следовательно, существует гомоморфизм $\psi: \mathfrak{A} / \vartheta(\mathfrak{Q}) \rightarrow G$ на некоторую ненулевую конечную абелеву группу $G$. Определим отображение $f: X_{A} \rightarrow G$ :

$$
f(x)=\psi(\bar{x}+\vartheta(\mathfrak{B})), \forall x \in X_{A} .
$$

Так как множество $\bar{X}$ - система образующих элементов группы $\mathfrak{A}$ и $\psi-$ эпиморфизм, то множество $f\left(X_{A}\right)$ - система образующих элементов группы $G$.

Как и ранее, отображение (41) определяет подгруппу $G^{(f)}$ группы $G$. Докажем равенство

$$
G^{(f)}=\left\{e_{G}\right\} .
$$

Из соотношений (4), (41) и (35) (учитывая линейность отображений $\psi, \vartheta)$ для любых таких $s, t \in S_{A}$, что $\delta_{A}(s, x)=t$ при некотором $x \in X_{A}$, следует цепочка равенств

$$
\begin{aligned}
\Omega\left(e_{s, t}^{+1}\right)=\{f(x), & \left.\delta_{A}(s, x)=t\right\}=\left\{f\left(\bar{x}_{s, t}\right)\right\}=\left\{\psi\left(\bar{x}_{s, t}+\vartheta(\mathfrak{B})\right)\right\}= \\
=\left\{\psi\left(\vartheta\left(\mathfrak{e}_{s, t}+\mathfrak{B}\right)\right)\right\} & =\left\{\psi\left(\vartheta\left(\phi\left(e_{s, t}^{+1}\right)+\mathfrak{B}\right)\right)\right\}
\end{aligned}
$$

и, аналогично,

$$
\begin{array}{r}
\Omega\left(e_{t, s}^{-1}\right)=\left\{-f(x), \delta_{A}(s, x)=t\right\}=\left\{-f\left(\bar{x}_{s, t}\right)\right\}=\left\{\psi\left(-\bar{x}_{s, t}+\vartheta(\mathfrak{B})\right)\right\}= \\
=\left\{\psi\left(\vartheta\left(-\mathfrak{e}_{s, t}+\mathfrak{P}\right)\right)\right\}=\left\{\psi\left(\vartheta\left(\phi\left(e_{t, s}^{-1}\right)+\mathfrak{B}\right)\right)\right\} .
\end{array}
$$

Используя соотношения (5), (33), (43), (44), (34), получаем цепочку равенств

$$
\Omega(z)=\psi(\vartheta(\phi(z)+\mathfrak{B}))=\{\psi(\vartheta(\mathfrak{B}))\}=\left\{e_{G}\right\} \quad \forall z \in Z_{0} .
$$

Из последнего равенства и соотношения (6) следует равенство (42).

Таким образом, по теореме 1 отображение (41) определяет нужный нам нетривиальный гомоморфизм автомата $A$. Теорема 3 доказана полностью.

Проверка соотношения (36) может быть проведена следующим образом. Аналогично доказательству следствия 2 из теоремы 1 можно показать, что (конечное) множество

$$
\Phi=\left\{\phi\left(u_{s, t}\right) \mid s, t \in S_{A}, \exists x \in X_{A}: \delta_{A}(s, x)=t\right\} \subseteq \mathfrak{f}
$$

является системой образующих элементов группы $\mathfrak{B}$. Следовательно, множество $\vartheta(\Phi) \subseteq \mathfrak{A}$ является системой образующих элементов группы $\vartheta(\mathfrak{B})$. 
Упорядочим некоторым образом элементы множества $\bar{X}$ :

$$
\bar{X}=\left\{\bar{x}_{1}, \bar{x}_{2}, \ldots, \bar{x}_{n}\right\}, \quad n=|\bar{X}|,
$$

а также элементы множества $\vartheta(\Phi)$ :

$$
\vartheta(\Phi)=\left\{\mathfrak{l}_{1}, \mathfrak{l}_{2}, \ldots, \mathfrak{l}_{m}\right\}, \quad m=|\vartheta(\Phi)|, \quad m \geq n .
$$

Для некоторых $c_{i, j} \in \mathbb{Z}, i \in\{1, \ldots, m\}, j \in\{1, \ldots, n\}$, верны равенства

$$
\mathfrak{l}_{i}=c_{i, 1} \cdot \bar{x}_{1}+c_{i, 2} \cdot \bar{x}_{2}+\ldots+c_{i, n} \cdot \bar{x}_{n} \quad \forall i \in\{1, \ldots, m\} .
$$

Рассмотрим целочисленную матрицу $C=\left[c_{i, j}\right]_{i=1, \ldots, m, j=1, \ldots, n}$ с $m$ строками и $n$ столбцами. Матрицу $C$ элементарными преобразованиями строк можно привести к целочисленному ступенчатому виду $C_{1}$ с $r$ ступеньками, $1 \leq r \leq n$.

Соотношение (36) выполнено в двух случаях: либо $r<n$, либо $r=n$, и матрица $C_{1}$ имеет вид

$$
C_{1}=\left(\begin{array}{ccccccc}
\gamma_{1} & \gamma_{1,2} & \ldots & \ldots & \ldots & \gamma_{1, n} & \\
0 & \gamma_{2} & \gamma_{2,3} & \ldots & \ldots & \gamma_{2, n} & \\
0 & 0 & \gamma_{3} & \gamma_{3,4} & \ldots & \gamma_{3, n} & \\
\vdots & \vdots & \vdots & \ddots & \vdots & \vdots & \vdots \\
0 & 0 & \ldots & 0 & \gamma_{n-1} & \gamma_{n-1, n} & \\
0 & 0 & \ldots & 0 & 0 & \gamma_{n} & \\
0 & 0 & 0 & 0 & 0 & 0 & \\
& \ldots & \ldots & \ldots & \ldots & \ldots & \ldots
\end{array}\right)_{m \times n}
$$

где $\gamma_{i} \neq 0$ при любом $i \in\{1, \ldots, n\}$ и $\left|\gamma_{i}\right|>1$ для некоторого $i \in\{1, \ldots, n\}$.

Автор считает своим приятным долгом поблагодарить В.И. Солодовникова за ряд ценных замечаний, в частности, за указание на наличие изоморфизма групп $G_{B}$ и $G_{B^{\prime}}$ в формулировке теоремы 2.

\section{Список литературы}

1. Бахтурин Ю.А. Основные структуры современной алгебры. - М.: Наука, 1990. $320 \mathrm{c}$.

2. Брауэр В. Введение в теорию конечных автоматов: Пер. с нем. - М.: Радио и связь, 1987. 392 с.

3. Сачков В.Н. Вероятностные преобразователи и правильные мультиграфы. 1. - В сб.: Труды по дискретной математике. Т. 1. - М.: ТВП, 1997, c. $227-250$. 